\title{
Catalog of Chromium, Cobalt, and Nickel Abundances in Globular Clusters and Dwarf Galaxies*
}

\author{
Evan N. Kirby ${ }^{1}$ (D), Justin L. Xie ${ }^{1,2}$, Rachel Guo ${ }^{1,3}$, Mikhail Kovalev ${ }^{4}$, and Maria Bergemann ${ }^{4}$ (iD \\ ${ }^{1}$ California Institute of Technology, 1200 E. California Blvd., MC 249-17, Pasadena, CA 91125, USA; enk@astro.caltech.edu \\ 2 The Harker School, 500 Saratoga Ave., San Jose, CA 95129, USA \\ 3 Irvington High School, 41800 Blacow Rd, Fremont, CA 94538, USA \\ ${ }^{4}$ Max-Planck Institute for Astronomy, D-69117, Heidelberg, Germany \\ Received 2018 April 2; revised 2018 May 23; accepted 2018 May 28; published 2018 July 19
}

\begin{abstract}
We present measurements of the abundances of chromium, cobalt, and nickel in 4113 red giants, including 2277 stars in globular clusters (GCs), 1820 stars in the Milky Way's dwarf satellite galaxies, and 16 field stars. We measured the abundances from mostly archival Keck/DEIMOS medium-resolution spectroscopy with a resolving power of $R \sim 6500$ and a wavelength range of approximately 6500-9000 $\AA$. The abundances were determined by fitting spectral regions that contain absorption lines of the elements under consideration. We used estimates of temperature, surface gravity, and metallicity that we previously determined from the same spectra. We estimated systematic error by examining the dispersion of abundances within mono-metallic GCs. The median uncertainties for $[\mathrm{Cr} / \mathrm{Fe}],[\mathrm{Co} / \mathrm{Fe}]$, and $[\mathrm{Ni} / \mathrm{Fe}]$ are $0.20,0.20$, and 0.13 , respectively. Finally, we validated our estimations of uncertainty through duplicate measurements, and we evaluated the accuracy and precision of our measurements through comparison to high-resolution spectroscopic measurements of the same stars.
\end{abstract}

Key words: galaxies: abundances - galaxies: dwarf - Local Group - nuclear reactions, nucleosynthesis, abundances - supernovae

Supporting material: machine-readable tables

\section{Introduction}

The iron group consists of elements close to iron $(Z=26)$ in the periodic table, and it is generally accepted to encompass the elements from chromium $(Z=24)$ to nickel $(Z=28)$. These elements form a local maximum in the cosmic abundance distribution, peaking at iron. The root cause of the local maximum is that these elements have high binding energies per nucleon relative to the adjacent elements. As a result, it is energetically favorable to create these elements in environments with sufficiently high temperature and density.

Different types of fusion create elements from different parts of the periodic table. Whereas hydrostatic burning generates most of the lighter elements, explosive burning creates elements in the iron peak (i.a., Woosley \& Weaver 1995). Therefore, supernovae are the sites of iron-peak nucleosynthesis. In fact, thermonuclear (Type Ia) supernovae are so efficient at generating iron-peak elements that their optical light curves are dominated by the radioactive decay of nickel and cobalt (Arnett 1969). Type Ia supernovae generate large amounts of ${ }^{56} \mathrm{Ni}$, which is the nuclide with the highest binding energy per nucleon. That nuclide radioactively decays into ${ }^{56} \mathrm{Co}$, which in turn decays into ${ }^{56} \mathrm{Fe}$.

Abundances of iron-peak elements can probe the physics of explosive nucleosynthesis. For example, the thermonuclear explosions of massive white dwarfs would be expected to generate relatively large amounts of neutron-rich nuclides compared to the explosions of less massive white dwarfs. The reason is that massive white dwarfs are very dense. High

\footnotetext{
* The data presented herein were obtained at the W.M. Keck Observatory, which is operated as a scientific partnership among the California Institute of Technology, the University of California and the National Aeronautics and Space Administration. The Observatory was made possible by the generous financial support of the W. M. Keck Foundation.
}

densities, coupled with electron degeneracy, favor the production of neutrons. Therefore, white dwarfs that explode at or near the Chandrasekhar mass would be expected to produce plenty of neutron-rich nuclides, including stable manganese (e.g., Seitenzahl et al. 2013), which has only one stable isotope: ${ }^{55} \mathrm{Mn}$ and ${ }^{59} \mathrm{Co}$. This isotope is quite neutron-rich. Therefore, detecting large amounts of $\mathrm{Mn}$ in long-lived stars might indicate that the Type Ia supernovae that contributed to the abundances of these stars exploded at or near the Chandrasekhar mass.

This article addresses the abundances of iron-peak elements in and around the Milky Way. We measure these abundances in mostly archival spectra of red giants, described in Section 2 . Nearly all of the giants in our sample are members of dwarf satellite galaxies or globular clusters (GCs). As described in Section 3, our innovation is to use medium-resolution spectroscopy rather than high-resolution spectroscopy, which allows us to analyze a sample size of thousands of stars. Because our measurements are not based on a traditional equivalent width analysis, we devote some time to the estimation of uncertainties (Section 4) and to the validation of our measurements through duplicate measurements and comparison to high-resolution spectroscopy in the literature (Section 5). Section 6 summarizes this article.

\section{Observations}

We use medium-resolution spectroscopy to measure abundances of iron-peak elements in stars in dwarf galaxies, GCs, and in the field of the Milky Way halo. The spectra come from the Keck Deep Imaging Multi-Object Spectrograph (DEIMOS, Faber et al. 2003). The vast majority of the data in this paper is archival, but we also obtained a few new spectra. 
Table 1

New DEIMOS Observations

\begin{tabular}{|c|c|c|c|c|c|c|c|}
\hline Target & Slitmask & Targets & UT Date & Airmass & Seeing $\left({ }^{\prime \prime}\right)$ & Exposures & Exp. Time (s) \\
\hline \multirow[t]{2}{*}{ NGC 4590} & $4590 c-1$ & 94 & 2016 Jan 29 & 1.5 & $\ldots$ & 3 & 3600 \\
\hline & $4590 c-2$ & 94 & 2016 May 11 & 1.5 & 1.2 & 2 & 2340 \\
\hline \multirow[t]{2}{*}{ NGC 5053} & n5053r-1 & 120 & 2016 Jan 29 & 1.5 & $\ldots$ & 3 & 3600 \\
\hline & n5053r-2 & 120 & 2016 Mar 5 & 1.0 & 0.5 & 3 & 3600 \\
\hline \multirow[t]{3}{*}{ NGC 5272} & $5272 \mathrm{c} 2$ & 134 & 2016 Dec 29 & 1.1 & 0.7 & 1 & 520 \\
\hline & n5272f-1 & 167 & 2016 Jan 29 & 1.2 & $\ldots$ & 3 & 3600 \\
\hline & $\mathrm{n} 5272 \mathrm{f}-2$ & 167 & 2016 May 11 & 1.1 & 0.7 & 3 & 3600 \\
\hline \multirow[t]{2}{*}{ NGC 5897} & 5897 ar-1 & 123 & 2016 Jan 29 & 1.4 & $\ldots$ & 3 & 3540 \\
\hline & 5897 ar- 2 & 123 & 2016 May 11 & 1.4 & 1.0 & 3 & 3600 \\
\hline Palomar 14 & pal14a & 40 & 2011 Aug 6 & 1.2 & 1.2 & 3 & 3960 \\
\hline \multirow[t]{2}{*}{ Sextans } & sexmil & 74 & 2010 May 11 & 1.1 & 0.5 & 3 & 3600 \\
\hline & sexmi4 & 76 & 2010 May 12 & 1.3 & 0.7 & 3 & 3600 \\
\hline \multirow[t]{9}{*}{ Ursa Minor } & umima1 & 122 & 2010 May 11 & 1.5 & 0.8 & 3 & 3600 \\
\hline & umima2 & 81 & 2010 May 11 & 1.5 & 0.6 & 3 & 3600 \\
\hline & umima3 & 64 & 2010 May 11 & 1.5 & 0.7 & 3 & 3600 \\
\hline & umimil & 87 & 2010 May 12 & 1.5 & 0.6 & 3 & 3600 \\
\hline & umimi2 & 76 & 2010 May 12 & 1.5 & 0.7 & 3 & 3600 \\
\hline & umimi3 & 67 & 2010 May 12 & 1.5 & 0.8 & 3 & 3600 \\
\hline & umimx 1 & 83 & 2010 May 11 & 1.6 & 0.8 & 3 & 3600 \\
\hline & umimx 2 & 81 & 2010 May $11-12$ & 1.8 & 0.9 & 2 & 2700 \\
\hline & umimx 4 & 135 & 2010 May 12 & 1.6 & 0.8 & 3 & 3600 \\
\hline
\end{tabular}

\subsection{Archival Spectra}

We rely primarily on the DEIMOS spectra from Kirby et al. (2010, henceforth called K10). K10 observed 2961 red giants in 8 dwarf galaxies, 445 red giants in $12 \mathrm{GCs}$, and 21 field giants in the Milky Way halo. Our sample also includes eight dwarf galaxies observed by Simon \& Geha (2007). Kirby et al. (2008b, 2013) used those spectra to measure atmospheric parameters like temperature and metallicity. We also include the spectra of 2227 red giants in 25 GCs observed by Kirby et al. (2016, henceforth called K16). Some of those spectra overlap with K10's sample.

All of the spectra were obtained with DEIMOS via custom slitmasks for the dwarf galaxies and GCs and via a longslit mask for the halo stars. DEIMOS was configured with the $1200 \mathrm{G}$ grating positioned with a central wavelength of $7800 \AA$. The grating has a groove spacing of $1200 \mathrm{~mm}^{-1}$ and a blaze wavelength of $7760 \AA$. It provides a roughly constant dispersion of $1.2 \AA$, which corresponds to a resolving power of $R \sim 6500$ at the central wavelength. The wavelength range was approximately $6500-9000 \AA$, but the exact range varied from spectrum to spectrum depending on the location of the slit within the slitmask. The OG550 filter was used to block light from the second and higher diffraction orders.

Flat-fielding was accomplished with a Qz lamp projected onto the telescope dome. Wavelength calibration was based on exposures of $\mathrm{Ne}, \mathrm{Ar}, \mathrm{Kr}$, and $\mathrm{Xe}$ arc lamps turned on simultaneously. The images were reduced with the spec $2 \mathrm{~d}$ pipeline (Cooper et al. 2012; Newman et al. 2013). The pipeline provides one-dimensional spectra that are flat-fielded, wavelength-calibrated, and background-subtracted.

\subsection{New Spectra}

We also obtained new spectra with DEIMOS in five GCs, as well as two dwarf galaxies. Table 1 gives the observing log for these new spectra. Most of the new GC data are repeat observations of the same stars observed by K16. These data were obtained in preparation for a study on the binarity of stars in GCs (E. N. Kirby et al. 2018, in preparation). We use the spectra in this work simply because they are available. Some of these masks were observed on two different dates. Table 1 identifies these separate observations with the suffix 1 or 2 . We also obtained new spectra in Sextans and Ursa Minor. The slitmasks were placed along the minor axis of Sextans. Hence, they are called "sexmi." In Ursa Minor, the slitmasks were placed along the major and minor axes ("umima" and "umimi"), as well as several other locations near the center of the galaxy ("umix").

The first four GCs in Table 1 were also observed by K16. The slitmask design was the same as described previously. To reiterate K16, the photometry was taken from P.B. Stetson, ${ }^{5}$ Walker (1994), Stetson (2000), Testa et al. (2001), and An et al. (2008). We designed the slitmask for Palomar 14 from Stetson's photometry and Saha et al.'s (2005) photometry. The dwarf galaxy slitmasks were designed with photometry from the Sloan Digital Sky Survey (Abazajian et al. 2009). Stars were selected to have the approximate colors and magnitudes of red giants at the distances of their respective galaxies. Theoretical isochrones (Girardi et al. 2004) were employed to help identify candidate stars in the color-magnitude diagram.

All of the observations were conducted with the same spectrographic configuration as described in Section 2.1. The two-dimensional spectra from the same night or adjacent nights were co-added together. However, spectra taken in different months were not co-added. They are listed on separate lines in Table 1.

K10 measured metallicities and some detailed abundance ratios of the stars in the sample described in Section 2.1, and we followed the same procedure for the new spectra. Part of that procedure involves determining the spectral continuum. Following the same procedure as K10, we estimated the $\mathrm{S} / \mathrm{N}$

\footnotetext{
5 Communicated privately and available at http://www2.cadc-ccda.hia-iha. nrc-cnrc.gc.ca/community/STETSON/standards/. See K16 for details.
} 
Table 2

Spectroscopic Targets

\begin{tabular}{|c|c|c|c|c|}
\hline Target & $N(\mathrm{Cr})$ & $N(\mathrm{Co})$ & $N(\mathrm{Ni})$ & $N$ (any) \\
\hline \multicolumn{5}{|c|}{ Dwarf Galaxies } \\
\hline Sculptor & 134 & 167 & 310 & 312 \\
\hline Fornax & 146 & 307 & 397 & 403 \\
\hline Ursa Major II & 0 & 1 & 2 & 3 \\
\hline Leo $\mathrm{T}$ & 0 & 0 & 3 & 3 \\
\hline Leo I & 179 & 320 & 560 & 562 \\
\hline Sextans & 19 & 14 & 45 & 46 \\
\hline Ursa Major I & 1 & 1 & 7 & 7 \\
\hline Leo II & 47 & 75 & 150 & 153 \\
\hline Leo IV & 0 & 0 & 1 & 1 \\
\hline Coma Berenices & 1 & 2 & 5 & 5 \\
\hline Canes Venatici II & 0 & 1 & 0 & 1 \\
\hline Canes Venatici I & 12 & 13 & 49 & 52 \\
\hline Ursa Minor & 48 & 33 & 143 & 146 \\
\hline Hercules & 2 & 1 & 4 & 4 \\
\hline Draco & 48 & 44 & 120 & 122 \\
\hline
\end{tabular}

\begin{tabular}{|c|c|c|c|c|}
\hline \multicolumn{5}{|c|}{ Globular Clusters } \\
\hline NGC 288 & 88 & 88 & 114 & 114 \\
\hline Palomar 2 & 2 & 5 & 8 & 9 \\
\hline NGC 1904 (M79) & 95 & 88 & 136 & 136 \\
\hline NGC 2419 & 43 & 45 & 74 & 74 \\
\hline NGC 4590 (M68) & 23 & 52 & 95 & 98 \\
\hline NGC 5024 (M53) & 16 & 19 & 41 & 41 \\
\hline NGC 5053 & 11 & 10 & 29 & 31 \\
\hline NGC $5272(\mathrm{M} 3)$ & 54 & 48 & 78 & 78 \\
\hline NGC 5634 & 34 & 37 & 65 & 65 \\
\hline NGC 5897 & 62 & 73 & 206 & 208 \\
\hline NGC 5904 (M5) & 53 & 50 & 56 & 56 \\
\hline Palomar 14 & 1 & 3 & 5 & 5 \\
\hline NGC 6205 (M13) & 48 & 37 & 69 & 69 \\
\hline NGC 6229 & 11 & 10 & 18 & 18 \\
\hline NGC 6341 (M92) & 32 & 44 & 114 & 124 \\
\hline NGC 6656 (M22) & 44 & 42 & 48 & 49 \\
\hline NGC 6779 (M56) & 34 & 29 & 57 & 57 \\
\hline NGC 6838 (M71) & 35 & 43 & 46 & 46 \\
\hline NGC 6864 (M75) & 72 & 70 & 125 & 126 \\
\hline NGC 7006 & 32 & 32 & 47 & 47 \\
\hline NGC 7078 (M15) & 99 & 159 & 294 & 300 \\
\hline NGC 7089 (M2) & 262 & 261 & 368 & 372 \\
\hline NGC 7099 (M30) & 28 & 45 & 123 & 125 \\
\hline Palomar 13 & 2 & 1 & 8 & 8 \\
\hline NGC 7492 & 10 & 7 & 21 & 21 \\
\hline
\end{tabular}

\begin{tabular}{lcccc}
\hline \multicolumn{5}{c}{ Field Stars } \\
\hline Milky Way halo & 8 & 8 & 15 & 16 \\
\hline \multicolumn{5}{c}{ Total } \\
\hline Total & 1836 & 2285 & 4056 & 4113 \\
\hline
\end{tabular}

per pixel as the median absolute deviation from the continuum in the spectral regions classified as continuum regions. To convert to $\mathrm{S} / \mathrm{N}$ per $\AA$, we multiplied by $\sqrt{0.33}$, where 0.33 is the number of A per pixel.

The elements previously measured by $\mathrm{K} 10$ are $\mathrm{Mg}, \mathrm{Si}, \mathrm{Ca}$, $\mathrm{Ti}$, and $\mathrm{Fe}$. In this work, we add $\mathrm{Cr}, \mathrm{Co}$, and $\mathrm{Ni}$ to the elemental abundances measured from these spectra. Table 2 lists the dwarf galaxies and GCs that make up the sample, along with the number of individual stars in each system with measurements of each of those three elements. Only stars with estimated uncertainties of less than 0.3 dex are included.

\subsection{Membership}

We classified stars as members or non-members of their respective clusters or galaxies on the basis of radial velocity and position in the color-magnitude diagram. For clusters, we additionally considered the metallicity of the star. K10 and K16 described membership determination in detail for dwarf galaxies and GCs, respectively. In brief, the stars were selected to be within three standard deviations of the mean radial velocity. Stars were eliminated if they were obviously nonmembers on the basis of their colors or magnitudes or if they had strong $\mathrm{NaI} 8190$ doublets, signaling that they are foreground dwarf stars. Finally, GC stars were selected to be within three standard deviations of the mean metallicity.

Many stars were observed multiple times. We use these repeat observations in Section 5.1 to validate our estimates of uncertainty. In all other contexts, we report the abundances for the spectrum with the highest $\mathrm{S} / \mathrm{N}$.

\section{Elemental Abundance Measurements}

Traditional abundance measurements with equivalent widths are not practical at the spectral resolution of DEIMOS. Many lines are blended together, making it unfeasible to disentangle the contribution to the line flux of one absorption line from another. Therefore, we use spectral synthesis.

K10 already measured atmospheric parameters and some elemental abundances for the stars in their sample. Because we are mostly using the same spectra, we simply adopted their parameters. We followed exactly the same procedure as K10 to derive the parameters for the new spectra described in Section 2.2. The atmospheric parameters are effective temperature $\left(T_{\text {eff }}\right)$, surface gravity $(\log g)$, metallicity $([\mathrm{Fe} / \mathrm{H}]),{ }^{6}$ and the $\alpha$-to-iron ratio $([\alpha / \mathrm{Fe}])$. The last parameter encapsulates the amount of various $\alpha$ elements, like $\mathrm{Mg}$, that are present in the atmosphere. This parameter has a secondary effect on the atmospheric structure of the star, largely through the presence of free electrons donated by $\alpha$ elements, especially $\mathrm{Mg}$ (VandenBerg et al. 2012). In addition to $\mathrm{Fe}$, individual elemental abundances of $\mathrm{Mg}, \mathrm{Si}, \mathrm{Ca}$, and $\mathrm{Ti}$ were also measured.

These atmospheric parameters and elemental abundances were measured via $\chi^{2}$ minimization between the observed spectra and a large grid of synthetic spectra. The grid was synthesized at $0.02 \AA$ resolution over the spectral range $6300-9100 \AA$ at a spacing of $\Delta T_{\text {eff }}=100 \mathrm{~K}, \Delta \log g=0.5, \Delta[\mathrm{Fe} / \mathrm{H}]=0.1$, and $\Delta[\alpha / \mathrm{Fe}]=0.1$. The spectra were continuum-normalized iteratively.

K10 used the atomic and molecular line list compiled by Kirby et al. (2008a). We used a subset of the same line list for the measurement of iron-peak abundances. Section 3.1 further describes the line list and our procedure for determining which lines to use.

We adopted the solar abundances of Asplund et al. (2009), except in the case of $\mathrm{Fe}$ and $\mathrm{Mg}$. We used $\epsilon(\mathrm{Fe})=12+$ $\log [n(\mathrm{Fe}) / n(\mathrm{H})]=7.52$ and $\epsilon(\mathrm{Mg})=7.58$ for consistency with K10. The adopted iron-peak solar abundances are $\epsilon(\mathrm{Cr})=5.64, \epsilon(\mathrm{Co})=4.99$, and $\epsilon(\mathrm{Ni})=6.22$.

For each star, we interpolated a synthetic spectrum from K10's grid based on the previously determined atmospheric parameters. This spectrum has the solar ratios of $[\mathrm{Cr} / \mathrm{Fe}]$,

\footnotetext{
6 We use the notation $[\mathrm{A} / \mathrm{B}]=\log [n(\mathrm{~A}) / n(\mathrm{~B})]-\log \left[n_{\odot}(\mathrm{A}) / n_{\odot}(\mathrm{B})\right]$, where $n(\mathrm{~A})$ is the number density of atom $\mathrm{A}$.
} 
Table 3

Catalog of Abundances

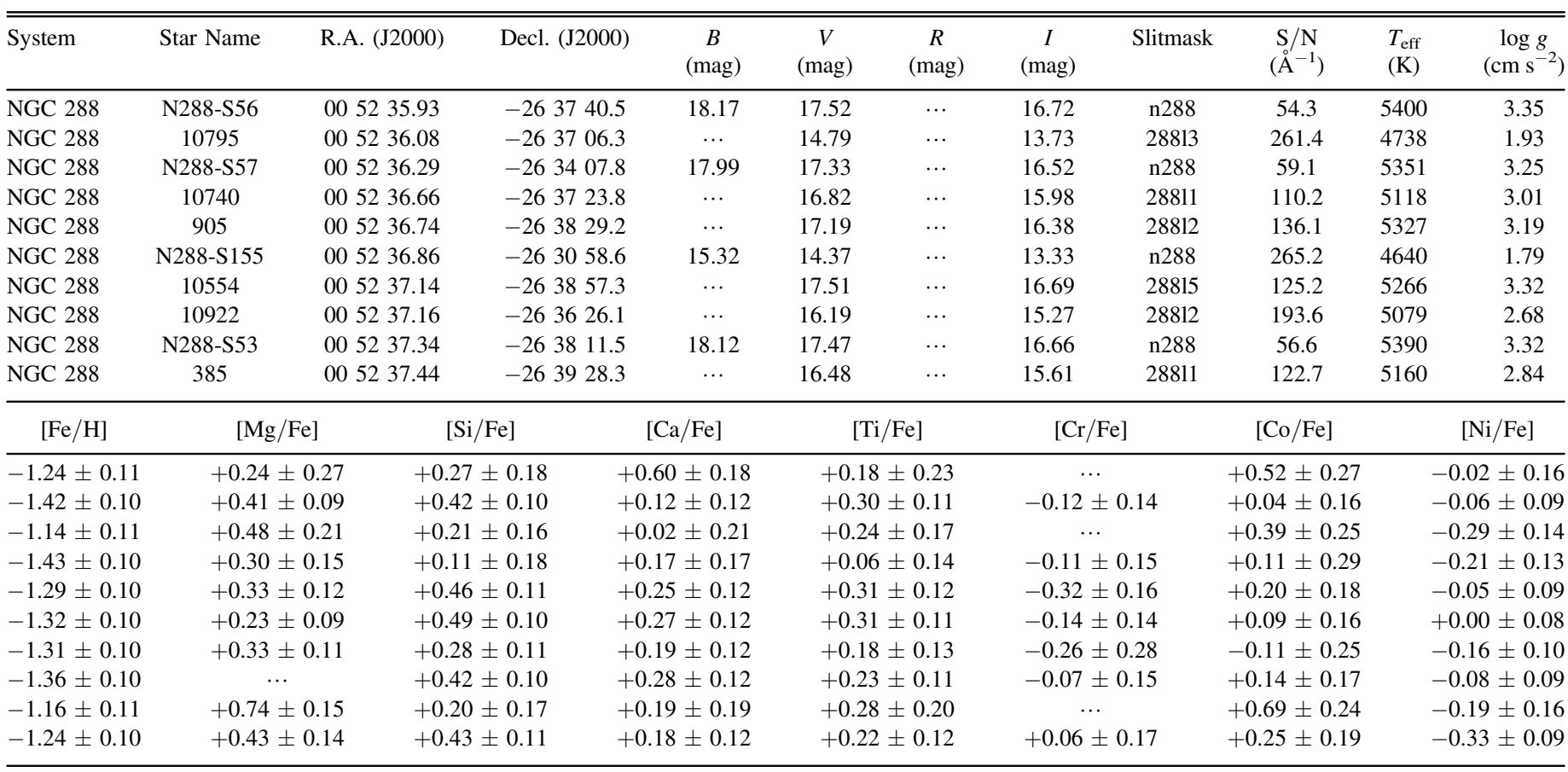

(This table is available in its entirety in machine-readable form.)

$[\mathrm{Co} / \mathrm{Fe}]$, and $[\mathrm{Ni} / \mathrm{Fe}]$. We call this spectrum $f_{\text {baseline }}\left(\lambda_{i}\right)$, where $i$ is the index of one pixel. The spectrum was smoothed to match the resolution of the DEIMOS spectrum. This spectrum was used to determine the continuum in the same manner as Kirby et al. (2009) and K10 by fitting a spline to the quotient of the observed spectrum and its corresponding model spectrum. The division removes the absorption lines and gives a mostly featureless spectrum, i.e., the continuum. We divided the observed spectrum by the continuum, yielding a spectrum normalized to unity between absorption lines.

In order to measure the abundances of iron-peak elements, we isolated the spectral regions sensitive to changes in the abundances of these elements, as described in Section 3.1. We synthesized spectra in these small regions, including a $2 \AA$ buffer on both sides of each region. We used the 2017 version of $\mathrm{MOOG}^{7}$ (Sneden 1973). MOOG is a spectral synthesis code that solves the radiative transfer equations in local thermodynamic equilibrium (LTE). We used the version of the code that was updated to include electron scattering in the calculation of the continuum opacity (Sobeck et al. 2011), coupled with plane parallel ATLAS9 (Kurucz 1993a) model atmospheres computed in LTE by Kirby (2011). These spectra are called $f_{\bmod }\left(\lambda_{j}\right)$, where $j$ represents the indices of pixels in the iron-peak regions. We then constructed new spectra, $f_{\text {merged }}\left(\lambda_{i}\right)$, where $f_{\text {merged }}\left(\lambda_{i}\right)=f_{\text {mod }}\left(\lambda_{j}\right)$ if $i \in j$ and $f_{\text {merged }}\left(\lambda_{i}\right)=f_{\text {baseline }}\left(\lambda_{i}\right)$ otherwise.

We measured elemental abundances by varying the input abundance in the computation of the spectra and calculating $\chi^{2}$ between the observed and synthetic spectra. This differs slightly from the approach of K10 in that we synthesized small regions of the spectrum on the fly rather than precomputing a grid of synthetic spectra. We found the minimum

7 Downloaded from https://github.com/alexji/moog17scat. $\chi^{2}$ with Levenberg-Marquardt minimization accomplished via the IDL routine MPFIT (Markwardt 2012). We quote the abundance corresponding to the minimum $\chi^{2}$ as the measured abundance. Table 3 gives the coordinates, photometric magnitudes, slitmask, $\mathrm{S} / \mathrm{N}$, temperature, gravity, and $\mathrm{Fe}, \mathrm{Mg}$, $\mathrm{Si}, \mathrm{Ca}, \mathrm{Ti}, \mathrm{Cr}, \mathrm{Co}$, and $\mathrm{Ni}$ abundances for each star in the sample. ${ }^{8}$ Abundances with uncertainty estimates greater than 0.3 dex (as determined in Section 4) are not shown.

\subsection{Iron-peak Line List}

The line list used for the computation of all model spectra in this work was taken from Kirby et al. (2008a), who compiled atomic and molecular information from the Vienna Atomic Line Database (VALD, Kupka et al. 1999), supplemented by molecular transitions (Kurucz 1992). Additionally, single-line atomic data for select elements, including $\mathrm{Co}$, were replaced by hyperfine blends (Kurucz 1993b). Kirby et al. (2008a) modified the oscillator strengths for some lines to match the spectra of the Sun and Arcturus.

Iron-peak elements other than iron do not affect the great majority of the pixels in the DEIMOS spectra. Therefore, we synthesized only those small regions sensitive to iron-peak abundances in order to save computation time. We chose these regions by constructing model spectra between $T_{\text {eff }}=3500 \mathrm{~K}$ and $8000 \mathrm{~K}$ at a spacing of $50 \mathrm{~K}$ and between $\log g=1.5$ and 3.5 at a spacing of 0.5 . The metallicity was $[\mathrm{Fe} / \mathrm{H}]=-1.0$ for all of the models, and the microturbulence was $\xi=$ 2.13-0.23 $(\log g)$ (Kirby et al. 2009). The model spectra were generated with MOOG and ATLAS9 model atmospheres, just as before. They were smoothed to match the DEIMOS spectral

\footnotetext{
8 In the course of preparing this article, we found a minor bug in the code that determines the spectral resolution of the DEIMOS spectrum. The abundances reported here were computed after fixing this bug. The typical change for any of the reported abundances is \pm 0.03 dex.
} 

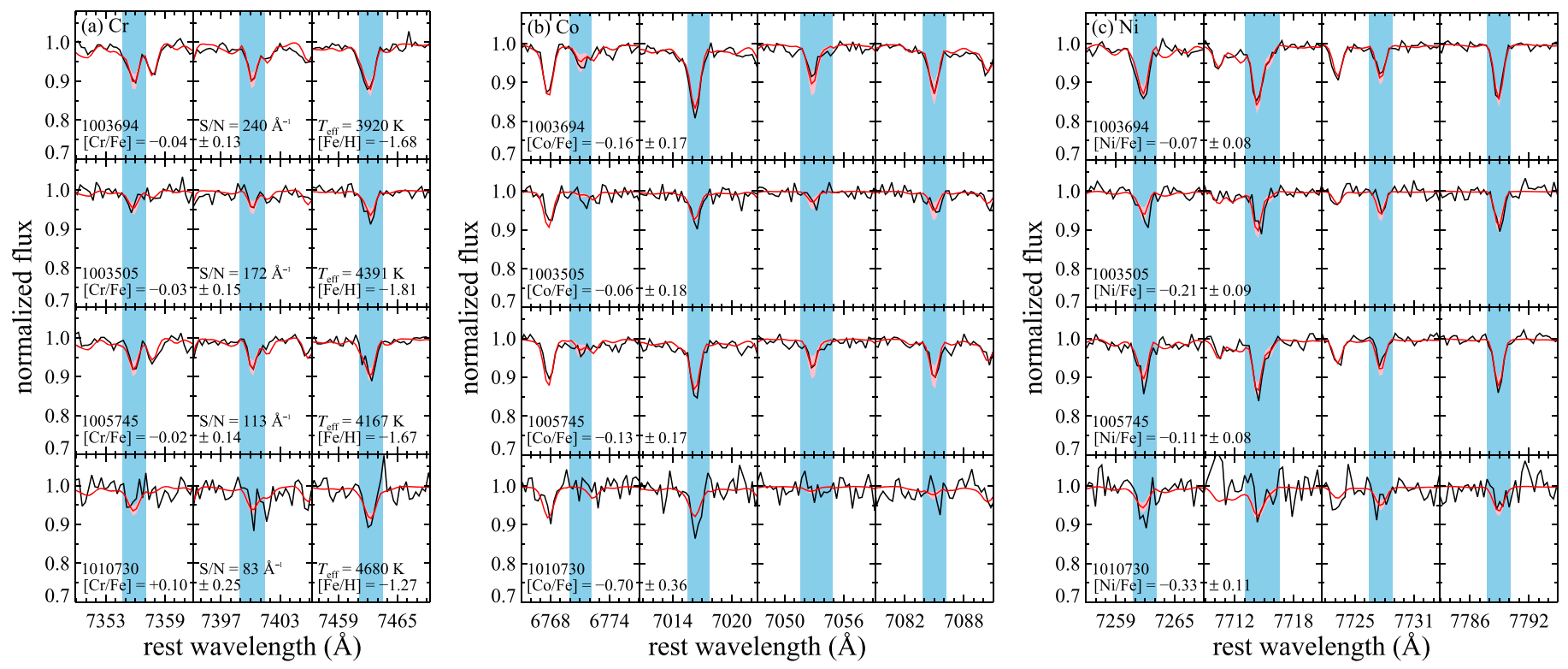

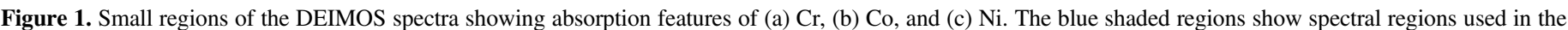

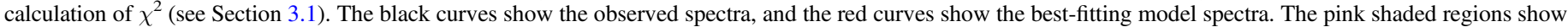

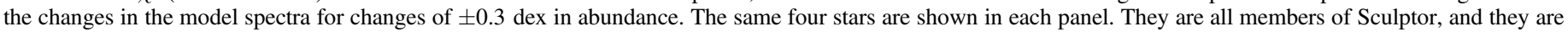

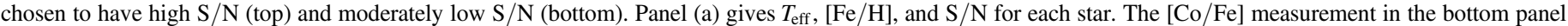

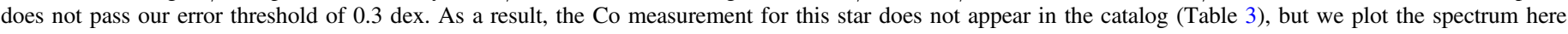
anyway.

Table 4

Important Atomic Lines

\begin{tabular}{lccc}
\hline \hline $\begin{array}{l}\text { Wavelength } \\
(\text { A, air })\end{array}$ & Species & $\begin{array}{c}\text { Excitation Potential } \\
(\mathrm{eV})\end{array}$ & $\log g f$ \\
\hline 7290.864 & Ni I & 5.342 & -0.235 \\
7291.453 & Ni I & 1.935 & -2.700 \\
7355.890 & Cr I & 2.890 & -0.100 \\
7393.600 & Ni I & 3.606 & -0.150 \\
7400.180 & Cr I & 2.900 & 0.050 \\
7409.346 & Ni I & 3.796 & -0.100 \\
7414.500 & Ni I & 1.986 & -2.300 \\
7417.267 & Co I & 2.042 & -2.900 \\
7417.330 & Co I & 2.042 & -2.500 \\
7417.350 & Co I & 2.042 & -3.100 \\
\hline
\end{tabular}

(This table is available in its entirety in machine-readable form.)

resolution (line profiles with $\sim 1.2 \AA$ FWHM). We separately analyzed the effect of the iron-group elements $\mathrm{Sc}, \mathrm{V}, \mathrm{Cr}, \mathrm{Mn}$, $\mathrm{Co}, \mathrm{Ni}, \mathrm{Cu}$, and $\mathrm{Zn}$ on the model spectra. (K10 already measured Ti.) For each element and each pair of $T_{\text {eff }}$ and $\log g$, we synthesized one spectrum with the solar ratio of the ironpeak element (e.g., $[\mathrm{Ni} / \mathrm{Fe}]=0$ ) and one spectrum without any of the iron-peak element (e.g., $[\mathrm{Ni} / \mathrm{Fe}]=-\infty$ ). We identified all the spectral regions of at least $0.75 \AA$ in width where the flux changed by at least $0.5 \%$ for any of the pairs of $T_{\text {eff }}$ and $\log g$. Finally, we examined each spectral region to compare model spectra to observed spectra with high $\mathrm{S} / \mathrm{N}$ to verify that the model was a reasonable representation of real spectra. We discarded a few spectral regions where the line strengths were obviously inconsistent between the model and observed spectra. The remaining spectral windows range in size from 1.1 to $4.5 \AA$.

Most elements did not have a large enough presence in the spectral region under consideration (approximately 6300-9100 $\mathrm{A}$ ) to be useful for measuring their abundances. Only $\mathrm{Cr}, \mathrm{Co}$, and $\mathrm{Ni}$ had enough spectral regions with sufficiently large responses to changes in abundance. The remaining discussion of iron-peak elements will be limited to these three elements. Sc, V, Mn, Cu, and $\mathrm{Zn}$ are excluded from the discussion.

Table 4 gives the $\mathrm{Cr}, \mathrm{Co}$, and $\mathrm{Ni}$ absorption lines in the spectral regions, including hyperfine structure for Co. There are $9 \mathrm{Cr}$ lines, 12 Co lines (140 when accounting for hyperfine structure), and $34 \mathrm{Ni}$ lines. The $\chi^{2}$ measurement for each element was performed only in the spectral regions sensitive to that element. The model spectra used for the computation of $\chi^{2}$ were computed with the full line list, not just the lines in Table 4 .

Figure 1 shows four examples of spectral regions for each of $\mathrm{Cr}, \mathrm{Co}$, and Ni. The number of spectral regions used exceeds those shown in the figure. The pink shading shows the sensitivity to abundance changes of \pm 0.3 dex. Although it may not seem that the spectra are particularly sensitive to \pm 0.3 dex changes, the full spectral range including all of the spectral regions has enough sensitivity to justify the uncertainties we quote.

\subsection{Non-LTE Corrections}

The assumption of LTE is sufficient for many applications. For example, iron abundances computed in LTE with one-dimensional atmospheres are typically different from non-LTE (NLTE) computations by $\lesssim 0.1$ dex (Bergemann et al. 2012). However, electronic transitions of some elements have larger NLTE corrections. Bergemann \& Cescutti (2010) and Bergemann et al. (2010) found that the NLTE corrections for $\mathrm{Cr}$ and $\mathrm{Co}$ are significant. Therefore, we explored the effect of including NLTE corrections on our measurements of $[\mathrm{Cr} / \mathrm{Fe}]$ and $[\mathrm{Co} / \mathrm{Fe}]$.

We computed corrections based on the formalism of Bergemann \& Cescutti (2010) and Bergemann et al. (2010), which we briefly describe here. The corrections were computed with two sets of model atmospheres: one-dimensional, plane 
parallel models generated with the MAFAGS-OS code (Grupp 2004a, 2004b) and one-dimensional, spherical models generated with the MARCS code (Gustafsson et al. 2008). We used the NLTE corrections based on the MARCS grid because they were computed in a temperature range that overlaps our sample better than the MAFAGS-OS grid. The corrections were computed on a grid of atmospheric parameters spanning $2500 \mathrm{~K} \leqslant T_{\text {eff }} \leqslant$ $7750 \mathrm{~K}, \quad-0.5 \leqslant \log g \leqslant 3.5$, and $-5.0 \leqslant[\mathrm{Fe} / \mathrm{H}] \leqslant 1.0$. Microturbulence was fixed at $\xi=2.13-0.23(\log g)$ (Kirby et al. 2009). An NLTE spectrum with $[\mathrm{Cr} / \mathrm{Fe}]=0$ or $[\mathrm{Co} / \mathrm{Fe}]=0$ was computed for each point on this grid for all $\mathrm{Cr}$ and Co transitions in Table 4. The syntheses were $10 \AA$ wide, centered on each line. Additionally, 21 LTE spectra were computed with abundance corrections ranging from $\Delta \epsilon=-1.0$ to 1.0 with a step size of 0.1 . Equivalent widths were computed for all spectra. The NLTE correction was taken to be the change in LTE abundance required to make the LTE equivalent width match the NLTE equivalent width. We performed linear interpolation in $\Delta \epsilon$ to minimize the difference in equivalent width between the LTE and NLTE spectra. These corrections are available in a queryable online database. ${ }^{9}$

Because the NLTE corrections are different for each transition, we cannot apply a single NLTE correction to the $\mathrm{Cr}$ or Co abundance of each star. Instead, we must apply separate NLTE corrections to the synthesis of each transition. We followed the same procedure described in Section 3, except we altered the oscillator strength $(\log g f)$ of each $\mathrm{Cr}$ or $\mathrm{Co}$ transition. We changed the oscillator strength by the abundance correction described in the previous paragraph. The effect of changing oscillator strength is identical to changing the input abundance. However, our approach allows us to compute synthetic spectra with multiple $\mathrm{Cr}$ or Co transitions, each with separate NLTE corrections. The new oscillator strength is $\log g f_{\text {mod, }, i}=\log g f_{\text {orig, }, i}-\Delta \epsilon_{i}$. In this equation, $\log g f_{\text {mod }, i}$ is the oscillator strength for line $i$ to be used as the input to an LTE code, like MOOG. Finally, $\Delta \epsilon_{i}$ is the NLTE correction for line $i$, which is a function of temperature, gravity, and metallicity. The sign convention is such that $\Delta \epsilon_{i}$ is the value that should be added to an LTE Co abundance measurement to give the NLTE abundance. This is the same convention on the NLTE website in footnote 9.

The resulting spectra had $9 \mathrm{Cr}$ and $12 \mathrm{Co}$ transitions. These were the model spectra that were used in the $\chi^{2}$ minimization. This procedure required us to sample NLTE corrections both between grid points and beyond the bounds of the NLTE corrections grid. We used linear interpolation and, where necessary, extrapolation.

Figure 2 shows the LTE and NLTE abundances for the GC M79 as a function of $T_{\text {eff }}$. We expect that the $\mathrm{Cr}, \mathrm{Fe}$, and Co abundances in M79 are constant. Therefore, they should not have a trend with $T_{\text {eff }}$. Any trend would indicate a systematic error in the abundances, possibly caused by the assumption of LTE. The LTE abundances for Cr show a U shape with $T_{\text {eff }}$, whereas the LTE abundances for Co rise by about 0.1 dex from $T_{\text {eff }}=4000$ to $5000 \mathrm{~K}$. However, the NLTE abundances increase strongly with $T_{\text {eff. }}$ The increase for $[\mathrm{Cr} / \mathrm{Fe}]_{\mathrm{NLTE}}$ is 0.5 dex from 4900 to $5400 \mathrm{~K}$, and the increase in $[\mathrm{Co} / \mathrm{Fe}]_{\mathrm{NLTE}}$ is 0.8 dex from 4200 to $5200 \mathrm{~K}$.

The strong trend of NLTE abundances with $T_{\text {eff }}$ indicates that our application of NLTE corrections does not lead to more

\footnotetext{
http://nlte.mpia.de
}

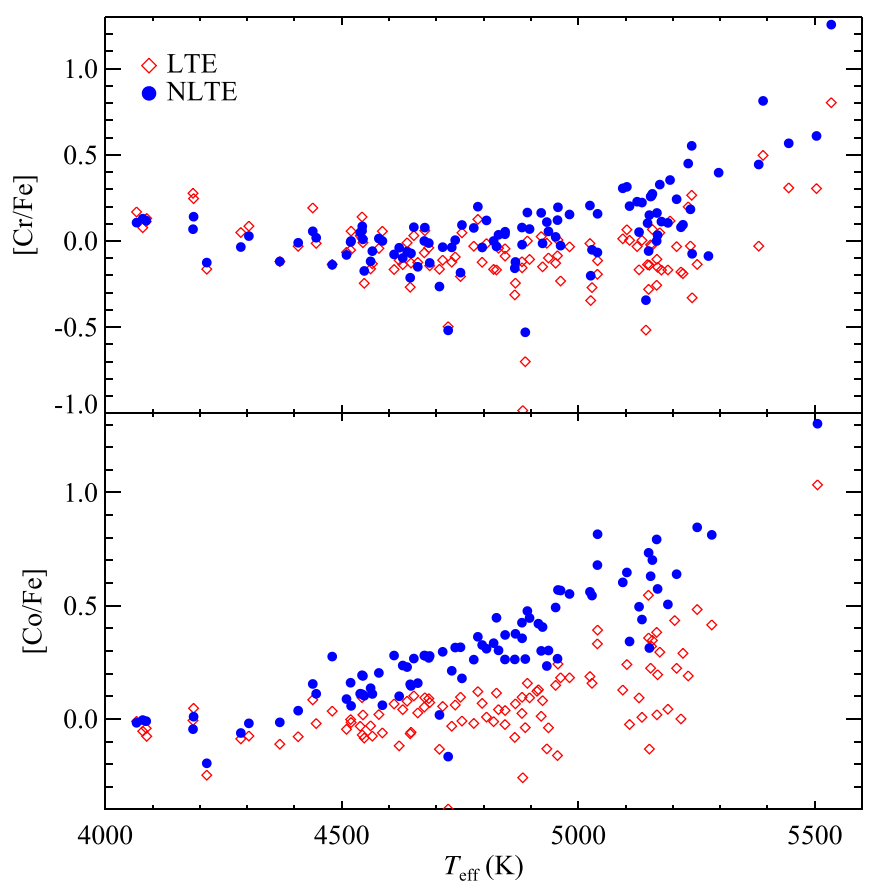

Figure 2. LTE and NLTE abundance ratios for $[\mathrm{Cr} / \mathrm{Fe}]$ and $[\mathrm{Co} / \mathrm{Fe}]$ as a function of effective temperature in the globular cluster M79.

accurate abundances. The cause for the lack of improvement is under investigation. One possibility is that the atmospheric parameters $\left(T_{\text {eff }}, \log g,[\mathrm{Fe} / \mathrm{H}]\right.$, and $\left.\xi\right)$ were computed assuming LTE. It is possible that the NLTE corrections are not appropriate for atmospheric parameters derived assuming LTE.

In summary, our investigation into NLTE corrections found that the corrections do not improve the abundance measurements. Therefore, we report only LTE abundances. The magnitude of the deviation of the LTE measurements of $[\mathrm{Cr} / \mathrm{Fe}]$ and $[\mathrm{Co} / \mathrm{Fe}]$ with $T_{\text {eff }}$ (Figure 2) provides one estimate for the magnitude of errors introduced by assuming LTE. In Section 4, we estimate the the bulk effect of systematic errors by quantifying the scatter of abundances within a GC. To the extent that assuming LTE increases this scatter, as shown in Figure 2, the error introduced by assuming LTE will be included in those estimates.

\section{Estimation of Uncertainties}

The uncertainty on the abundance ratios, $\delta[\mathrm{X} / \mathrm{Fe}]$, consists of two components: a random uncertainty from the spectral fitting and a systematic error. Our error model assumes that these components add in quadrature.

$$
\delta[\mathrm{X} / \mathrm{Fe}]=\sqrt{\delta_{\mathrm{fit}}[\mathrm{X} / \mathrm{Fe}]^{2}+\delta_{\mathrm{sys}}[\mathrm{X} / \mathrm{Fe}]^{2}} .
$$

MPFIT calculates the random uncertainty, $\delta_{\mathrm{fit}}[\mathrm{X} / \mathrm{Fe}]$, as the change in $[\mathrm{X} / \mathrm{Fe}]$ required to increase the unreduced $\chi^{2}$ by one.

We estimated the systematic error, $\delta_{\text {sys }}[\mathrm{X} / \mathrm{Fe}]$, in a manner similar to that of Kirby et al. (2008a). The method uses only stars in GCs. We assume that each $\mathrm{GC}$ has a single value of $[\mathrm{Cr} / \mathrm{Fe}]$, a single value of $[\mathrm{Co} / \mathrm{Fe}]$, and a single value of $[\mathrm{Ni} / \mathrm{Fe}]$. Any deviation in the abundance measurements of any one star from these values must be explained by the quoted uncertainty in the star. We accomplish this by computing a 

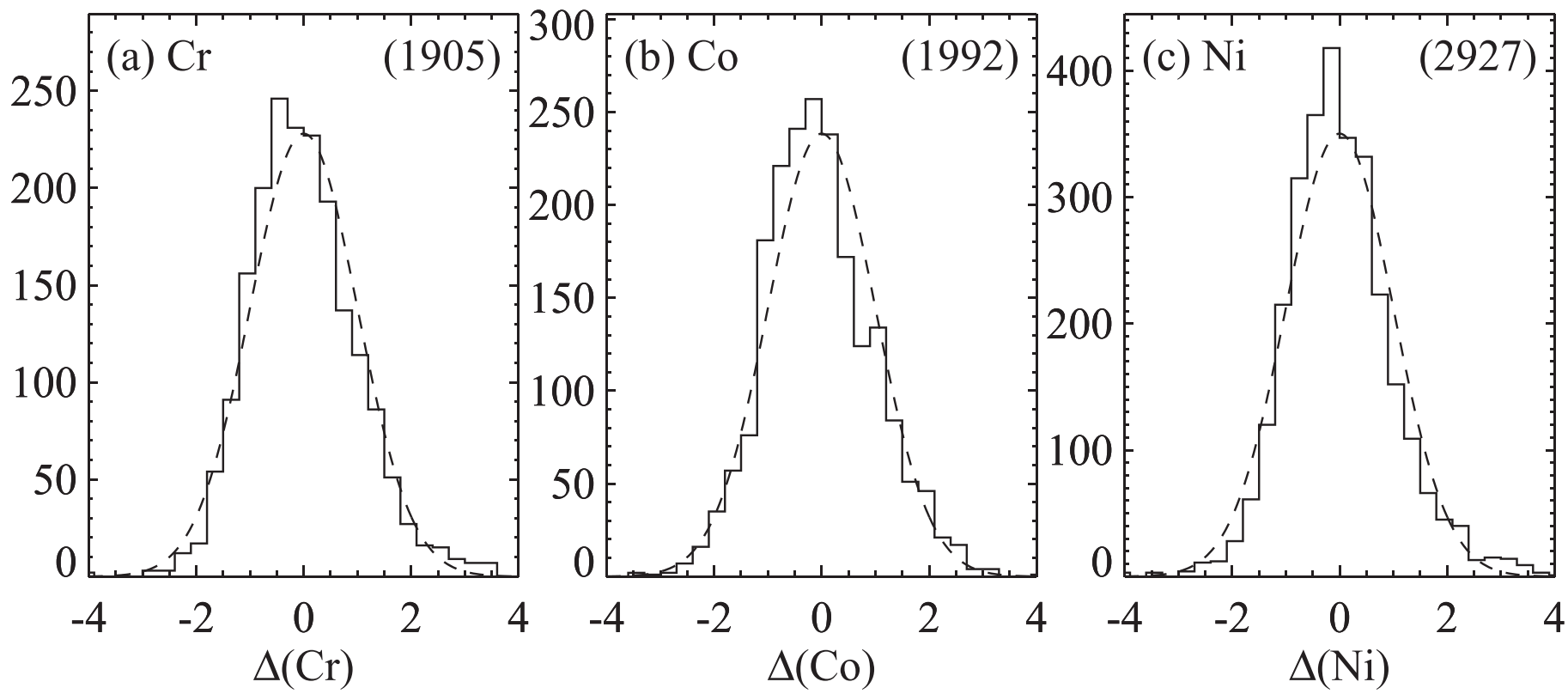

Figure 3. Distributions of $\Delta$ (Equation (2)) used in determining the systematic errors for the iron-peak abundance measurements. The dashed curves show Gaussians with a variance of unity. In the limit of perfectly Gaussian-distributed uncertainties, the histograms would conform to the dashed curves. The numbers of stars are indicated in parentheses.

value called $\Delta$ :

$$
\Delta_{i}=\frac{[\mathrm{X} / \mathrm{Fe}]_{i}-\langle[\mathrm{X} / \mathrm{Fe}]\rangle_{j}}{\sqrt{\delta_{\mathrm{fit}}[\mathrm{X} / \mathrm{Fe}]^{2}+\delta_{\mathrm{sys}}[\mathrm{X} / \mathrm{Fe}]^{2}}},
$$

where $i$ represents an individual star and $j$ represents the cluster to which that star belongs. The mean value for the $j$ th cluster, $\langle[\mathrm{X} / \mathrm{Fe}]\rangle_{j}$, is weighted by the inverse variances of the measurements of $[\mathrm{X} / \mathrm{Fe}]$.

We expect $\Delta$ to obey a Gaussian distribution with a variance of unity if the uncertainties on $[\mathrm{X} / \mathrm{Fe}]$ are properly estimated and Gaussian-distributed. Therefore, we determine $\delta_{\text {sys }}[\mathrm{X} / \mathrm{Fe}]$ by finding the value that gives $\Delta$ a variance of unity. The corresponding systematic errors for $[\mathrm{Cr} / \mathrm{Fe}],[\mathrm{Co} / \mathrm{Fe}]$, and $[\mathrm{Ni} / \mathrm{Fe}]$, respectively, are $0.123,0.169$, and 0.077 .

Figure 3 shows the distribution of $\Delta$ for these values of $\delta_{\text {sys }}[\mathrm{X} / \mathrm{Fe}]$. The uncertainties shown in Table 3 are derived from Equation (1). The table includes only those stars with uncertainties less than 0.3 dex. The median uncertainties are $0.20,0.20$, and 0.13 for $[\mathrm{Cr} / \mathrm{Fe}],[\mathrm{Co} / \mathrm{Fe}]$, and $[\mathrm{Ni} / \mathrm{Fe}]$, respectively.

Our estimate of the systematic error is predicated on the assumption that the element ratios have no dispersion within GCs. This assumption might be challenged by our inclusion of M2 and M22, which are GCs that have been suggested to display intrinsic dispersions in their iron abundances (Marino et al. 2009; Yong et al. 2014). However, there is no evidence that either cluster shows a dispersion in the ratios of an ironpeak element to iron, such as $[\mathrm{Ni} / \mathrm{Fe}]$. Furthermore, the claimed dispersion in iron has been challenged in both cases as a side effect of using spectroscopic rather than photometric surface gravities (Mucciarelli et al. 2015; Lardo et al. 2016). In any case, we experimented by determining $\delta_{\mathrm{sys}}[\mathrm{X} / \mathrm{Fe}]$, excluding M2 and M22. The resulting values of $\delta_{\text {sys }}[\mathrm{X} / \mathrm{Fe}]$ actually increased by $0.003-0.008$ dex, even though we would expect them to decrease if the clusters had intrinsic dispersions in iron-peak abundance ratios. Therefore, we kept these clusters in the sample.

\section{Validation}

We validate our measurements in two ways. First, we quantify the consistency among separate measurements of the same star. In this way, we assess the validity of the estimated uncertainties. Second, we compare our measurements to previously published high-resolution spectroscopic abundance measurements of the same stars in order to assess both accuracy and precision.

\subsection{Consistency Among Repeated Observations}

One way to assess the resilience of our measurements to both spectral noise and certain systematic errors is to examine repeat measurements of the same star. Our sample contains 311, 339, and 760 duplicate measurements of $[\mathrm{Cr} / \mathrm{Fe}],[\mathrm{Co} / \mathrm{Fe}]$, and $[\mathrm{Ni} / \mathrm{Fe}]$, respectively. These numbers include all the $\left(\begin{array}{l}N \\ 2\end{array}\right)$ permutations for every star that was observed $N$ times.

We quantify the consistency among these repeat measurements in a manner similar to Equation (2). For each pair of observations $i$ and $j$, we compute the quantity $\delta_{i j}$ :

$$
\delta_{i j}=\frac{[\mathrm{X} / \mathrm{Fe}]_{i}-[\mathrm{X} / \mathrm{Fe}]_{j}}{\sqrt{\delta_{\mathrm{fit}}[\mathrm{X} / \mathrm{Fe}]_{i}^{2}+\delta_{\mathrm{fit}}[\mathrm{X} / \mathrm{Fe}]_{j}^{2}+\delta_{\mathrm{sys}}[\mathrm{X} / \mathrm{Fe}]^{2}}} .
$$

The denominator includes only one factor of the systematic error term, even though $\delta_{i j}$ is computed from the observations of two stars. We made this choice because some of the systematic error comes from variables, such as the photometry, that are common to both measurements. In a more sophisticated analysis, the systematic error term would have a coefficient that encapsulates the degree of dissimilarity between the variables that enter into the computation of the abundance measurements for the pair of spectra.

Figure 4 shows the distributions of $\delta_{i j}$ for each element. In the limit of perfectly determined abundances and perfectly estimated uncertainties, the distribution of $\delta_{i j}$ would have a 

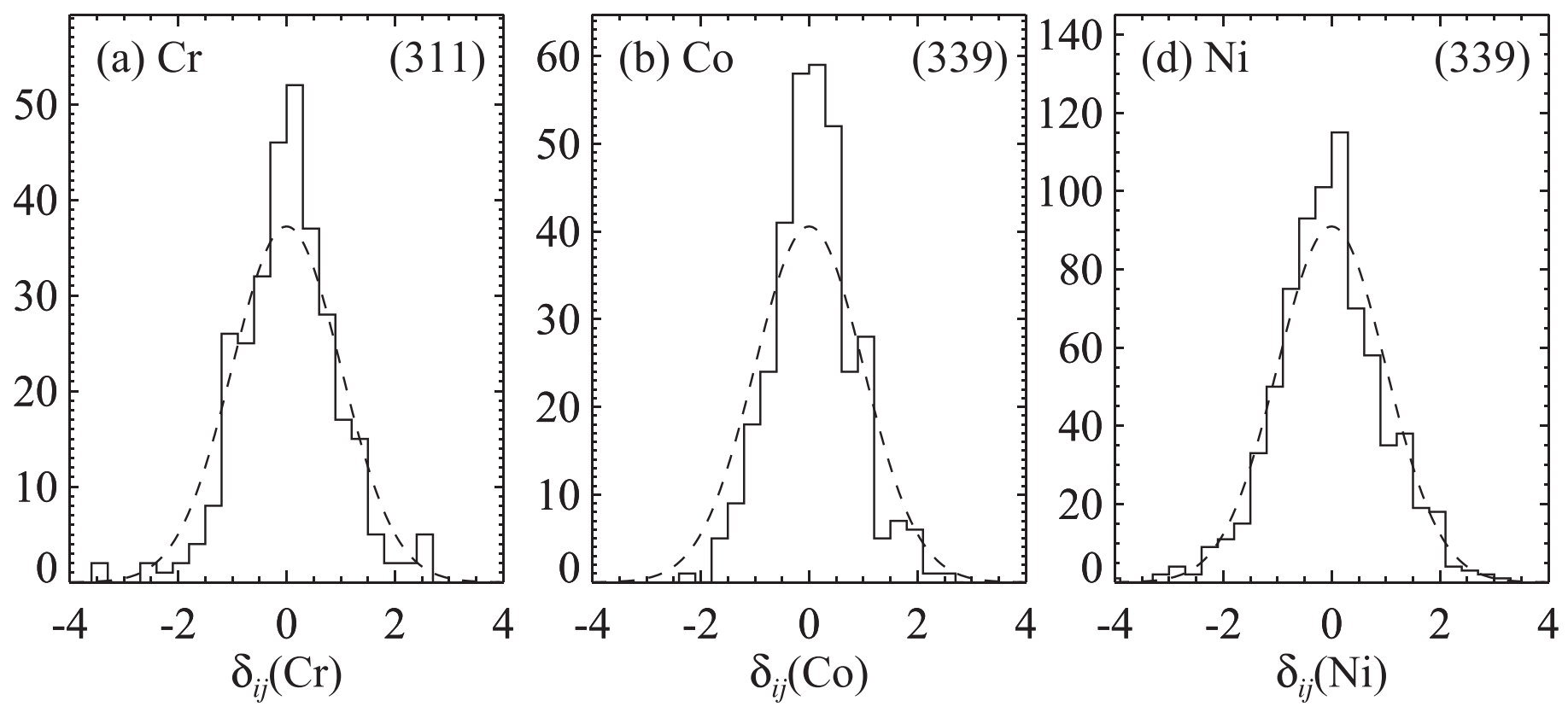

Figure 4. Differences in measured abundance ratios between repeated observations of the same stars. The differences are normalized by the quadrature sum of the uncertainties (Equation (3)). The dashed curves show Gaussians with zero mean and unit variance. Each panel gives the number of pairs of measurements in parentheses.

mean of zero and a variance of unity, as represented by the dashed curves. The actual mean values of $\delta_{i j}$ are $0.03 \pm 0.06$, $0.08 \pm 0.05$, and $-0.05 \pm 0.04$ for $\mathrm{Cr}, \mathrm{Co}$, and $\mathrm{Ni}$, respectively. In reality, these mean values are not meaningful because the stars $i$ and $j$ in Equation (3) can be swapped arbitrarily.

The standard deviations of the $\delta_{i j}$ are $0.90 \pm 0.04$, $0.76 \pm 0.03$, and $0.99 \pm 0.03$ for $\mathrm{Cr}, \mathrm{Co}$, and $\mathrm{Ni}$, respectively. These values indicate that we have estimated $\delta_{\text {sys }}[\mathrm{Cr} / \mathrm{Fe}]$ and $\delta_{\text {sys }}[\mathrm{Ni} / \mathrm{Fe}]$ well, but we have perhaps overestimated $\delta_{\text {sys }}[\mathrm{Co} / \mathrm{Fe}]$. It could even be the case that Co abundances are variable within GCs. Our estimation of uncertainties (Section 4) assumes that each GC has a single Co abundance. If it is variable, then $\delta_{\text {sys }}[\mathrm{Co} / \mathrm{Fe}]$ will encompass that variability. Alternatively, the denominator in Equation (3) might not properly account for the systematic error for duplicate measurements, as discussed above. For example, there seems to be a slight trend of abundance with $T_{\text {eff }}$ (Figure 2). This systematic trend would not be represented by $\delta_{i j}$ because the repeat measurements have similar values of $T_{\text {eff }}$. In either case, the errors do not appear to be underestimated.

\subsection{Comparison to High-resolution Spectroscopic Abundances}

We validated our medium-resolution spectroscopic (MRS) abundance measurements by comparing them to high-resolution spectroscopic (HRS) abundance measurements for the same stars, where available. The comparison sample largely overlaps with the HRS sample compiled from the literature by K10 (see their Table 7). Some stars from K10 are not included either because we could not measure iron-peak abundances from the DEIMOS spectra or because the literature source for the HRS did not list those values. We also supplemented the comparison set with measurements from ultra-faint galaxies (Simon \& Geha 2007; Kirby et al. 2008b, 2013) and additional GCs (K16). The comparison sample includes 128 stars: 59 stars in GCs, 9 field stars in the Milky Way's metal-poor halo, and 60 stars in dwarf galaxies. We shifted all abundances onto our adopted solar abundance scale. None of the literature references applied NLTE corrections to Cr or Co abundances. We also use the element-to-iron ratios rather than the absolute abundances (e.g., $[\mathrm{Cr} / \mathrm{Fe}]$ rather than $\epsilon(\mathrm{Cr}))$ because the ratio removes most of the effect of differences in atmospheric parameters, especially $T_{\text {eff. }}$ Also, the abundance ratio will be the more interesting quantity for our future analyses in the context of galactic chemical evolution.

Table 5 gives the MRS and HRS measurements of iron-peak abundances for the stars in common. In cases of repeated MRS measurements, the table includes multiple entries for the same star. For those entries, the HRS abundances are identical, but different MRS measurements are given on different lines. Figure 5 shows the differences between our measurements and the HRS measurements from the literature. The mean differences $\left(\left\langle[\mathrm{X} / \mathrm{Fe}]_{\mathrm{MRS}}-[\mathrm{X} / \mathrm{Fe}]_{\mathrm{HRS}}\right\rangle\right)$ weighted by the quadrature sum of the HRS and MRS uncertainties are $+0.11 \pm 0.03$ for $\mathrm{Cr}$, $-0.01 \pm 0.04$ for $\mathrm{Co}$, and $+0.01 \pm 0.01$ for $\mathrm{Ni}$. The differences are negligible for $\mathrm{Co}$ and $\mathrm{Ni}$. The difference for $\mathrm{Cr}$ is small enough not to cause concern. It could arise from a number of factors, but we point out that the large majority of our DEIMOS spectra include only three of the $\mathrm{Cr}$ absorption lines listed in Table 4. All three of these lines are red enough that they are seldom included in HRS spectroscopic studies. Thus, the difference might be a result of using a different set of Cr lines.

If both the MRS and HRS measurements are accurate and their uncertainties are properly estimated, then we expect the differences normalized by the quadrature sum of their uncertainties to be normally distributed with a mean of zero and standard deviation of unity

$$
\operatorname{mean}\left(\frac{[\mathrm{X} / \mathrm{Fe}]_{\mathrm{MRS}, i}-[\mathrm{X} / \mathrm{Fe}]_{\mathrm{HRS}, i}}{\sqrt{\delta[\mathrm{X} / \mathrm{Fe}]_{\mathrm{MRS}}^{2}+\delta[\mathrm{X} / \mathrm{Fe}]_{\mathrm{HRS}}^{2}}}\right)=0
$$



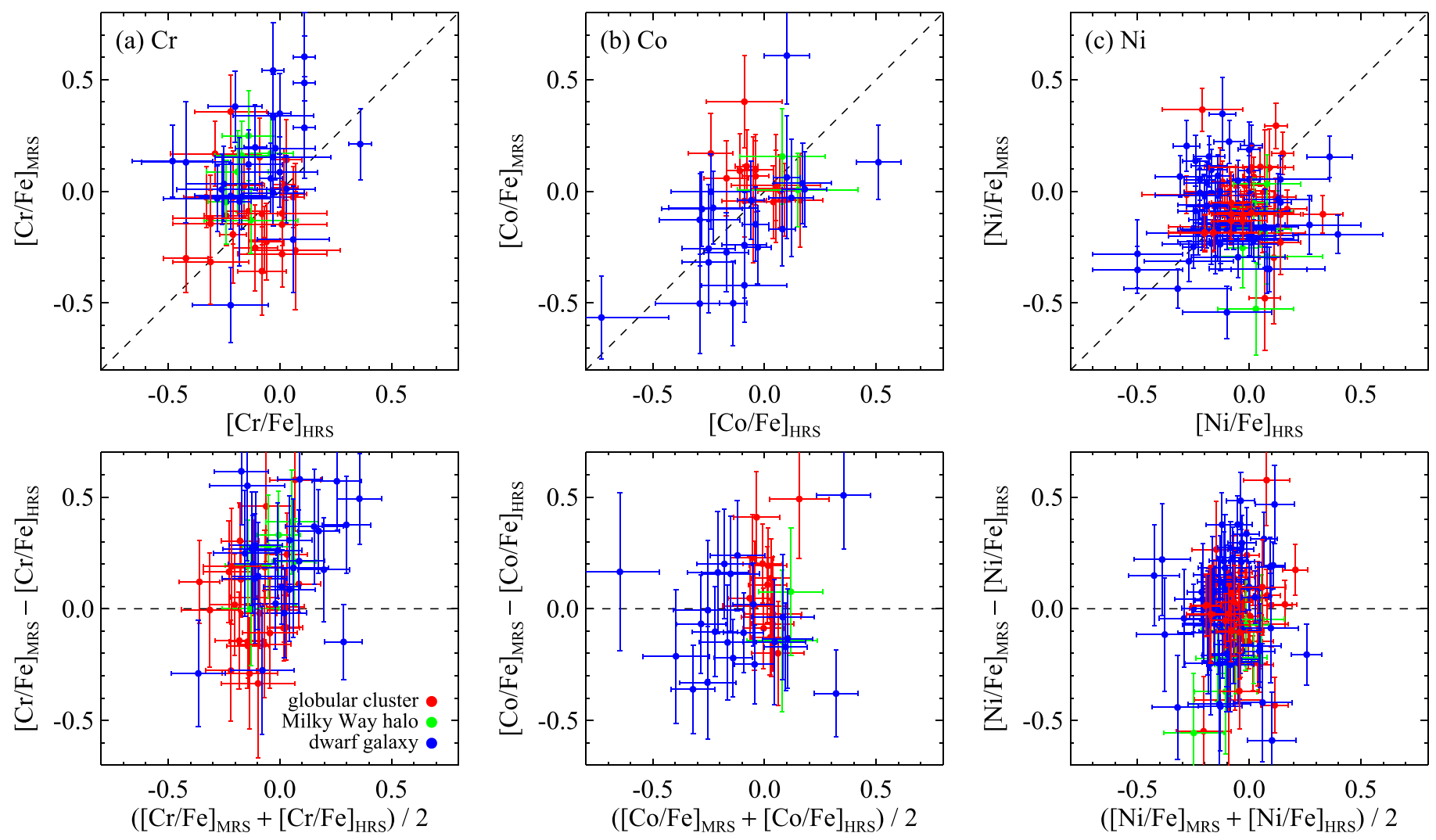

Figure 5. Comparison between our MRS measurements and HRS measurements from the literature for the same stars for (a) $[\mathrm{Cr} / \mathrm{Fe}],(b)[\mathrm{Co} / \mathrm{Fe}]$, and (c) $[\mathrm{Ni} / \mathrm{Fe}]$. Color-coding indicates the type of stellar system in which the stars reside. The dashed lines indicate one-to-one agreement.

Table 5

Comparison to High-resolution Spectroscopic Abundances

\begin{tabular}{|c|c|c|c|c|c|}
\hline System & & Star Name & & & HRS Reference \\
\hline M68 & & $\mathrm{I}-256$ & & & Gratton \& Ortolani (1989) \\
\hline M5 & & III-149 & & & Ivans et al. (2001) \\
\hline M5 & & G18155_0228 & & & Ramírez \& Cohen (2003) \\
\hline M5 & & $1-36$ & & & Ramírez \& Cohen (2003) \\
\hline M5 & & $\mathrm{I}-71$ & & & Ivans et al. (2001) \\
\hline M5 & & II-59 & & & Ivans et al. (2001) \\
\hline M5 & & $\mathrm{I}-58$ & & & Ivans et al. (2001) \\
\hline M5 & & G18447_0453 & & & Ramírez \& Cohen (2003) \\
\hline M5 & & $1-31$ & & & Ramírez \& Cohen (2003) \\
\hline M5 & & IV-59 & & & Ivans et al. (2001) \\
\hline$[\mathrm{Cr} / \mathrm{Fe}]_{\mathrm{HRS}}$ & {$[\mathrm{Co} / \mathrm{Fe}]_{\mathrm{HRS}}$} & {$[\mathrm{Ni} / \mathrm{Fe}]_{\text {HRS }}$} & {$[\mathrm{Cr} / \mathrm{Fe}]_{\mathrm{MRS}}$} & {$[\mathrm{Co} / \mathrm{Fe}]_{\mathrm{MRS}}$} & {$[\mathrm{Ni} / \mathrm{Fe}]_{\mathrm{MRS}}$} \\
\hline$+0.01 \pm 0.20$ & $\ldots$ & $-0.16 \pm 0.20$ & $-0.10 \pm 0.14$ & 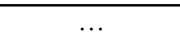 & $-0.18 \pm 0.08$ \\
\hline$\ldots$ & $\ldots$ & $-0.06 \pm 0.03$ & $\ldots$ & $\ldots$ & $-0.07 \pm 0.09$ \\
\hline$\ldots$ & $\ldots$ & $-0.14 \pm 0.15$ & $\ldots$ & $\ldots$ & $-0.00 \pm 0.11$ \\
\hline$+0.07 \pm 0.20$ & $+0.05 \pm 0.11$ & $-0.09 \pm 0.05$ & $-0.26 \pm 0.26$ & $+0.01 \pm 0.24$ & $-0.00 \pm 0.11$ \\
\hline$\ldots$ & $\ldots$ & $-0.08 \pm 0.08$ & $\ldots$ & $\ldots$ & $-0.08 \pm 0.10$ \\
\hline$\ldots$ & $\ldots$ & $-0.06 \pm 0.04$ & $\ldots$ & $\ldots$ & $+0.03 \pm 0.09$ \\
\hline$\ldots$ & $\ldots$ & $-0.02 \pm 0.11$ & $\ldots$ & $\ldots$ & $+0.03 \pm 0.09$ \\
\hline$-0.42 \pm 0.10$ & $\ldots$ & $+0.06 \pm 0.19$ & $-0.30 \pm 0.15$ & $\ldots$ & $-0.19 \pm 0.09$ \\
\hline$-0.08 \pm 0.07$ & $-0.09 \pm 0.10$ & $-0.10 \pm 0.04$ & $-0.10 \pm 0.14$ & $-0.04 \pm 0.16$ & $-0.10 \pm 0.08$ \\
\hline$\ldots$ & $\ldots$ & $-0.13 \pm 0.07$ & $\ldots$ & $\ldots$ & $+0.11 \pm 0.09$ \\
\hline
\end{tabular}

(This table is available in its entirety in machine-readable form.)

$$
\operatorname{stddev}\left(\frac{[\mathrm{X} / \mathrm{Fe}]_{\mathrm{MRS}, i}-[\mathrm{X} / \mathrm{Fe}]_{\mathrm{HRS}, i}}{\sqrt{\delta[\mathrm{X} / \mathrm{Fe}]_{\mathrm{MRS}}^{2}+\delta[\mathrm{X} / \mathrm{Fe}]_{\mathrm{HRS}}^{2}}}\right)=1 .
$$

In fact, the means are $+0.57 \pm 0.16,-0.01 \pm 0.16$, and $-0.10 \pm 0.13$ for $\mathrm{Cr}, \mathrm{Co}$, and $\mathrm{Ni}$, respectively. The standard deviations are $1.14 \pm 0.11,1.00 \pm 0.11$, and $1.41 \pm 0.09$. 


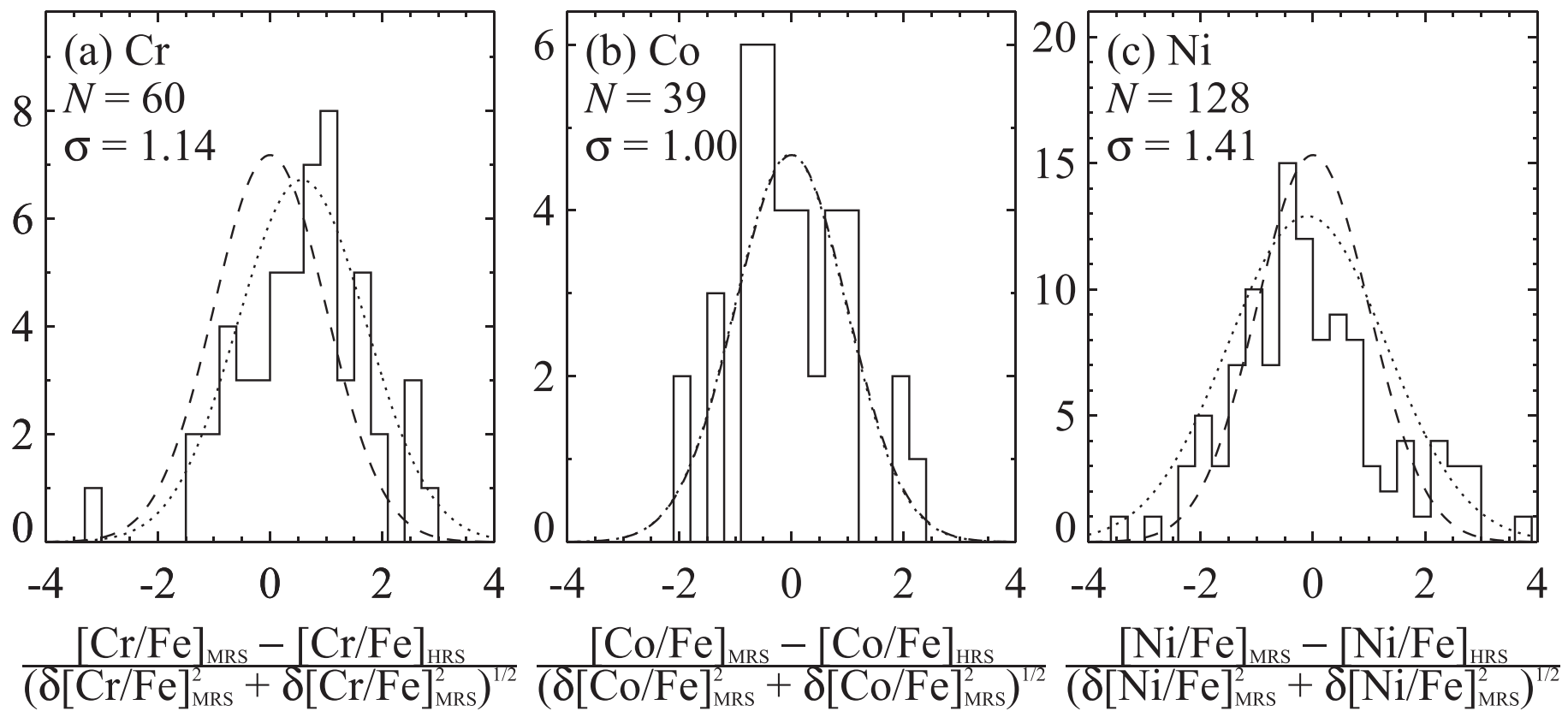

Figure 6. Differences between our abundance measurements from MRS and literature values from HRS for the same stars. The differences are normalized by the quadrature sum of the uncertainties. If the errors are Gaussian and properly estimated, then these histograms would be Gaussians with a standard deviation of 1 , as shown in Equation (5). The dashed curves show Gaussians with $\sigma=1$, whereas the dotted curves show the Gaussians that best represent the histograms. Each panel gives the number of stars and actual standard deviations for each comparison sample.

Figure 6 shows the distributions of the quantity in parentheses in Equations (4) and (5). The distributions for $\mathrm{Cr}$ and $\mathrm{Co}$ do indeed appear approximately Gaussian with standard deviations near 1 . The distribution for Ni is positively skewed and leptokurtic, and its formal standard deviation significantly exceeds 1 . These differences indicate systematic differences between MRS and HRS in the measurement of $\mathrm{Ni}$ and in the estimation of uncertainties.

Such differences are not surprising, given the large degree of heterogeneity among the comparison sample, which is comprised of 23 different literature sources, most of which have different line lists, abundance codes, and model atmospheres. Hinkel et al. (2016) showed that the results of even HRS spectroscopic analyses depend sensitively on the choice of these various inputs. (We also refer to Suda et al. 2017 for a comparison of different methods of determining abundances in dwarf galaxies.) Thus, the apparent imperfections shown in Figure 6(c) should not be taken as an indication of serious problems in the abundance measurements.

\section{Summary}

The abundances of iron-peak elements trace the components of galactic chemical evolution that are driven by explosive nucleosynthesis. These abundances are especially sensitive to the explosion properties of Type Ia supernovae. We have measured abundances of the iron-peak elements $\mathrm{Cr}, \mathrm{Co}$, and $\mathrm{Ni}$ for 4113 metal-poor red giants in GCs, dwarf galaxies, and the Milky Way halo. We used mostly archival and some new Keck/DEIMOS spectra, which we modeled with LTE synthetic spectra. Table 3 presents the catalog of measurements, including previously measured abundances of $\mathrm{Mg}, \mathrm{Si}, \mathrm{Ca}, \mathrm{Ti}$, and $\mathrm{Fe}(\mathrm{K} 10)$ and the new measurements of $\mathrm{Cr}, \mathrm{Co}$, and $\mathrm{Ni}$.

We estimated systematic errors by enforcing that the abundance of each element is constant within individual GCs. The total uncertainty is the quadrature sum of a fitting error that depends on $\mathrm{S} / \mathrm{N}$ and the systematic error. We validated the appropriateness of the uncertainty estimates by quantifying the dispersion of differences between repeat measurements of the same stars. The results indicated that the $\mathrm{Cr}$ and $\mathrm{Ni}$ uncertainties are well-determined, but the $\mathrm{Co}$ uncertainties might be slightly overestimated, at least at a given effective temperature. We also validated the accuracy and precision of our measurements by comparing them to highresolution spectroscopic measurements of the same stars. The results are that our $[\mathrm{Cr} / \mathrm{Fe}]$ measurements are $(+0.11 \pm 0.03)$ dex higher than the high-resolution literature values, and our $[\mathrm{Ni} / \mathrm{Fe}]$ measurements are slightly more discrepant with the literature values than indicated by our uncertainty estimates. Otherwise, the measurements agree within expectations.

Our analysis does not reveal any serious concerns regarding the accuracy of our measurements or our ability to assess the uncertainties. Thus, we have proven the capability to measure $\mathrm{Cr}$, Co, and $\mathrm{Ni}$ abundances from $R \sim 6500$ spectra in the wavelength range 6500-9000 $\AA$. The major advantage of our data set is its homogeneity. The spectra were obtained on the same instrument in the same configuration, and we measured the abundances with the same software. This represents the largest database of self-consistent multi-element abundance measurements in both GCs and dwarf galaxies.

We attempted to apply NLTE corrections to our abundances, but we we found that the corrections introduced trends in abundance with effective temperature. A better method of accounting for NLTE effects is to compute a spectral grid in NLTE (see Ruchti et al. 2013). These calculations are underway, and we are planning to employ them in the future.

Future work also includes measuring $\mathrm{Mn}$, an iron-peak element that is especially useful in diagnosing the explosion mechanism of Type Ia supernovae (Seitenzahl et al. 2013). The wavelength range of the DEIMOS spectra presented in this article does not include enough $\mathrm{Mn}$ lines for a reliable measurement. We intend to obtain bluer spectra in the future to measure Mn abundances. 
This article has not addressed any scientific interpretation of our results. An article in preparation will analyze the abundances in the context of nucleosynthesis from various models of Type Ia supernovae.

We thank the anonymous referee for a constructive report. This material is based upon work supported by the National Science Foundation under grant No. AST-1614081. Support for this work was also provided by NASA through a grant (HST-GO-14734.011-A) from the Space Telescope Science Institute, which is operated by the Association of Universities for Research in Astronomy, Inc., under NASA contract NAS 5-26555. E.N.K. acknowledges funding from generous donors to the California Institute of Technology.

The data presented herein were obtained at the W. M. Keck Observatory, which is operated as a scientific partnership among the California Institute of Technology, the University of California and the National Aeronautics and Space Administration. The Observatory was made possible by the generous financial support of the W. M. Keck Foundation. The authors wish to recognize and acknowledge the very significant cultural role and reverence that the summit of Maunakea has always had within the indigenous Hawaiian community. We are most fortunate to have the opportunity to conduct observations from this mountain.

Software: MOOG (Sneden 1973), MAFAGS-OS (Grupp 2004a, 2004b), MARCS (Gustafsson et al. 2008), spec2d pipeline (Cooper et al. 2012; Newman et al. 2013), MPFIT (Markwardt 2012).

Facility: Keck:II (DEIMOS).

\section{ORCID iDs}

Evan N. Kirby (1) https://orcid.org/0000-0001-6196-5162 Maria Bergemann (i) https://orcid.org/0000-0002-9908-5571

\section{References}

Abazajian, K. N., Adelman-McCarthy, J. K., Agüeros, M. A., et al. 2009, ApJS, 182, 543

An, D., Johnson, J. A., Clem, J. L., et al. 2008, ApJS, 179, 326

Arnett, W. D. 1969, Ap\&SS, 5, 180

Asplund, M., Grevesse, N., Sauval, A. J., \& Scott, P. 2009, ARA\&A, 47, 481

Bergemann, M., \& Cescutti, G. 2010, A\&A, 522, A9

Bergemann, M., Lind, K., Collet, R., Magic, Z., \& Asplund, M. 2012, MNRAS, 427, 27

Bergemann, M., Pickering, J. C., \& Gehren, T. 2010, MNRAS, 401, 1334

Cohen, J. G., \& Huang, W. 2009, ApJ, 701, 1053

Cohen, J. G., \& Huang, W. 2010, ApJ, 719, 931

Cohen, J. G., \& Meléndez, J. 2005, AJ, 129, 303

Cohen, J. G., \& Melendez, J. 2005, AJ, 129, 1607

Cooper, M. C., Newman, J. A., Davis, M., Finkbeiner, D. P., \& Gerke, B. F. 2012, spec2d: DEEP2 DEIMOS Spectral Pipeline, Astrophysics Source Code Library, ascl:1203.003

Faber, S. M., Phillips, A. C., Kibrick, R. I., et al. 2003, Proc. SPIE, 4841, 1657 Frebel, A., Simon, J. D., Geha, M., \& Willman, B. 2010, ApJ, 708, 560
Fulbright, J. P. 2000, AJ, 120, 1841

Fulbright, J. P., Rich, R. M., \& Castro, S. 2004, ApJ, 612, 447

Geisler, D., Smith, V. V., Wallerstein, G., Gonzalez, G., \& Charbonnel, C. 2005, AJ, 129, 1428

Girardi, L., Grebel, E. K., Odenkirchen, M., \& Chiosi, C. 2004, A\&A, 422, 205

Gratton, R. G., \& Ortolani, S. 1989, A\&A, 211, 41

Grupp, F. 2004a, A\&A, 420, 289

Grupp, F. 2004b, A\&A, 426, 309

Gustafsson, B., Edvardsson, B., Eriksson, K., et al. 2008, A\&A, 486, 951

Hinkel, N. R., Young, P. A., Pagano, M. D., et al. 2016, ApJS, 226, 4

Ivans, I. I., Kraft, R. P., Sneden, C., et al. 2001, AJ, 122, 1438

Johnson, J. A. 2002, ApJS, 139, 219

Kirby, E. N. 2011, PASP, 123, 531

Kirby, E. N., Cohen, J. G., Guhathakurta, P., et al. 2013, ApJ, 779, 102

Kirby, E. N., Guhathakurta, P., Bolte, M., Sneden, C., \& Geha, M. C. 2009, ApJ, 705, 328

Kirby, E. N., Guhathakurta, P., Simon, J. D., et al. 2010, ApJS, 191, 352, K10

Kirby, E. N., Guhathakurta, P., \& Sneden, C. 2008a, ApJ, 682, 1217

Kirby, E. N., Guhathakurta, P., Zhang, A. J., et al. 2016, ApJ, 819, 135, K16

Kirby, E. N., Simon, J. D., Geha, M., Guhathakurta, P., \& Frebel, A. 2008b, ApJL, 685, L43

Koch, A., McWilliam, A., Grebel, E. K., Zucker, D. B., \& Belokurov, V. 2008, ApJL, 688, L13

Kraft, R. P., Sneden, C., Smith, G. H., Shetrone, M. D., \& Fulbright, J. 1998, AJ, 115, 1500

Kupka, F., Piskunov, N., Ryabchikova, T. A., Stempels, H. C., \& Weiss, W. W. 1999, A\&AS, 138, 119

Kurucz, R. 1993a, ATLAS9 Stellar Atmosphere Programs and $2 \mathrm{~km} / \mathrm{s}$ grid. Kurucz CD-ROM No. 13 (Cambridge, MA: Smithsonian Astrophysical Observatory), 13

Kurucz, R. L. 1992, RMxAA, 23, 45

Kurucz, R. L. 1993b, PhST, 47, 110

Lardo, C., Mucciarelli, A., \& Bastian, N. 2016, MNRAS, 457, 51

Lee, J.-W., Carney, B. W., \& Habgood, M. J. 2005, AJ, 129, 251

Letarte, B., Hill, V., Tolstoy, E., et al. 2010, A\&A, 523, A17

Marino, A. F., Milone, A. P., Piotto, G., et al. 2009, A\&A, 505, 1099

Markwardt, C. 2012, MPFIT: Robust non-linear Least Squares Curve Fitting, Astrophysics Source Code Library, ascl:1208.019

Mishenina, T. V., Panchuk, V. E., \& Samus', N. N. 2003, ARep, 47, 248

Mucciarelli, A., Bellazzini, M., Merle, T., et al. 2015, ApJ, 801, 68

Newman, J. A., Cooper, M. C., Davis, M., et al. 2013, ApJS, 208, 5

Ramírez, S. V., \& Cohen, J. G. 2002, AJ, 123, 3277

Ramírez, S. V., \& Cohen, J. G. 2003, AJ, 125, 224

Ruchti, G. R., Bergemann, M., Serenelli, A., Casagrande, L., \& Lind, K. 2013 , MNRAS, 429, 126

Sadakane, K., Arimoto, N., Ikuta, C., et al. 2004, PASJ, 56, 1041

Saha, A., Dolphin, A. E., Thim, F., \& Whitmore, B. 2005, PASP, 117, 37

Seitenzahl, I. R., Cescutti, G., Röpke, F. K., Ruiter, A. J., \& Pakmor, R. 2013, A\&A, 559, L5

Shetrone, M., Venn, K. A., Tolstoy, E., et al. 2003, AJ, 125, 684

Shetrone, M. D., Bolte, M., \& Stetson, P. B. 1998, AJ, 115, 1888

Shetrone, M. D., Côté, P., \& Sargent, W. L. W. 2001, ApJ, 548, 592

Simon, J. D., \& Geha, M. 2007, ApJ, 670, 313

Sneden, C., Kraft, R. P., Shetrone, M. D., et al. 1997, AJ, 114, 1964

Sneden, C. A. 1973, PhD thesis, Univ. Texas Austin

Sobeck, J. S., Kraft, R. P., Sneden, C., et al. 2011, AJ, 141, 175

Stetson, P. B. 2000, PASP, 112, 925

Suda, T., Hidaka, J., Aoki, W., et al. 2017, PASJ, 69, 76

Testa, V., Corsi, C. E., Andreuzzi, G., et al. 2001, AJ, 121, 916

VandenBerg, D. A., Bergbusch, P. A., Dotter, A., et al. 2012, ApJ, 755, 15

Walker, A. R. 1994, AJ, 108, 555

Woosley, S. E., \& Weaver, T. A. 1995, ApJS, 101, 181

Yong, D., Roederer, I. U., Grundahl, F., et al. 2014, MNRAS, 441, 3396 